\title{
DEGREES OF FREEDOM AND LAGRANGIANS EXPLICITLY DEPENDING ON THE WEYL TENSOR*
}

\author{
LESZEK M. SOKOŁOWSKI \\ Astronomical Observatory, Jagiellonian University \\ Orla 171, 30-244 Kraków, Poland \\ lech.sokolowski@uj.edu.pl \\ (Received December 21, 2016)
}

This is an introduction to the problem of how generic may be a Lagrangian replacing the $L=R$ one of Einstein's General Relativity. We show that if the Lagrangian explicitly depends on the Weyl tensor, it is difficult to assign degrees of freedom to the tensor. In a tenable theory of gravity, the Lagrangian should be free of the conformal tensor, only the Ricci tensor is admissible.

DOI:10.5506/APhysPolBSupp.10.365

The article is based on a forthcoming paper with Magnano [1] and deals with a problem of how generic may be a theory of gravitation belonging to a specific class of generalizations of Einstein's General Relativity. This problem may seem bizarre to the reader. For two questions immediately arise: is it possible at all to reject General Relativity and replace it by an alternative theory and, if possible, is it worth doing?

The answer to the first question is "yes, it is possible", because there is a multitude of gravity theories. Physics of gravitation is exceptional in that in all other branches of physics, there is one or at most a couple of competing theories, whereas for gravitation there is infinite number of existing or potential theories. All these alternative gravity theories are merely various modifications, generalizations and complications of Einstein's GR and without it, they would never arise. GR is very sensitive (unlike quantum mechanics) to various extensions and formally it is merely a point in a continuous space of conceivable theories which have developed out of it.

Why can these theories compete with GR in describing gravitational effects? The reason is purely empirical. The number of experiments and observations that are accessible to us is small and their variety is small (though

* Talk presented at the $3^{\text {rd }}$ Conference of the Polish Society on Relativity, Kraków, Poland, September 25-29, 2016. 
we are now far beyond "the three classical tests" of GR). Our knowledge of gravitation is purely passive: we can describe existing gravitational fields, yet we cannot actively operate with them. Just compare: we cannot produce in a controlled way gravitational effects in such a number and variety as we can do in electromagnetism. We cannot create strong gravitational waves or black holes. Apparently, gravitation is a macroscopic phenomenon related to large masses. In consequence, many disparate theories fit sufficiently well to the scarce set of empirical data.

Modifications of GR go in all possible directions and classes of alternative are disparate; they are so abundant that up to now they have not been classified. Yet a deeper investigation of the structure of the set of theories forming a large neighbourhood of GR in the space of gravity theories shows that GR is distinguished not only historically (as the seed), but by its mathematical relationships to its surrounding.

The alternative theories appeared soon after the advent of GR and are traced back to Hermann Weyl (ca. 1919). The recent vivid interest in them is due to the problem of apparent accelerated expansion of the universe. Therefore, the second question may be formulated more precisely as: why to employ some alternative theories instead of assuming that the cosmic acceleration is driven in the framework of GR by a kind of self-interacting scalar (or vector or tensor) matter field? The answer is that if it is dark energy that rules, then one must introduce ad hoc some classical field unknown to laboratory physics. This classical field (no particles) exerts negative pressure, does not fit the Standard Model and contradicts the tenet of modern physics that all matter fields are quantized. Yet the gravitational field is classical and need not be quantized (empirically, there are no grounds for it) and is independent from the Standard Model. Replacement of GR by an alternative theory is more conservative than the concept of dark energy.

Most investigated alternative theories are metric nonlinear gravity (NLG) theories, which differ from GR in one only axiom: the Einstein-Hilbert Lagrangian $L=R$ is replaced by $L=f\left(g_{\mu \nu}, R_{\alpha \beta \mu \nu}\right)$ with any smooth scalar $f$ (no derivatives of the Riemann tensor). We emphasize (though it is irrelevant to our present considerations) that all NLG theories encounter a hard problem: they are inherently ambiguous in physical interpretation due to inevitable field redefinitions and only GR is free of this ambiguity. The problem is either ignored or solved by an arbitrary assumption.

Here, we deal with NLG theories from purely theoretical viewpoint, without any resort to observations. Our investigation is based on the following tenet of field theory: each fundamental field has a definite nonnegative mass and spin; if a field in the Lagrangian has no concrete mass and spin, it is a unifying field (a mixture) for a number of distinct fundamental fields. For an NLG theory, the metric in $L=f\left(g_{\mu \nu}, R_{\alpha \beta \mu \nu}\right)$ turns out a unifying field 
and must be decomposed into a multiplet of gravitational fields. One of the multiplet fields is the spacetime metric, identical to $g_{\mu \nu}$ or not, and other fields in the multiplet have no geometrical sense. This means that an NLG theory is not identified with the geometry. Physically, the decomposition of $g_{\mu \nu}$ into the multiplet determines the particle content of the NLG theory. To establish the particle content, it is not necessary to explicitly quantize the gravitational theory, it is sufficient that the unifying metric may be decomposed into a multiplet of classical fields with definite masses and spins. The generic $L=f\left(g_{\mu \nu}, R_{\alpha \beta \mu \nu}\right)$ does not correspond to a well-defined multiplet of fundamental fields.

The method of decomposition is not given a priori (is not inherent to NLG theories), it must be independently introduced and is evaluated according to its outcome. Equations of motion for the multiplet must be equivalent to equations for the unifying metric. An important ingredient of checking the equivalence and studying properties of the multiplet fields is counting degrees of freedom (DOF) for the unifying metric and for the fields, since the two numbers must be equal. By the number of DOF, we mean one half of the number of independent Cauchy data (when all constraints are satisfied) for the system of field equations [2]. Here, we do not identify the DOF, we merely count them. For counting DOF, the Hamiltonian formalism (very intricate) applied in GR, is not necessary. The maximal possible number of DOF for a generic $L=f\left(g_{\mu \nu}, R_{\alpha \beta \mu \nu}\right)$ is $F=8$ ( $F=2$ for GR). To the best of our knowledge, the best decomposition method for $g_{\mu \nu}$ in NLG theories is a formalism of generalized Legendre transformations. We have no space here to present this formalism and we refer the reader to [1] and references therein. We state only that this formalism is covariant and is distinct from the standard Hamiltonian formalism, though it is akin to the latter in that it is based on the notion of a kind of Legendre transformations.

We stress that from the field theory viewpoint, an NLG theory is tenable only if it has a well-defined particle content. However, it is impossible to study in this aspect NLG theories in full generality. A generic $L=f\left(g_{\mu \nu}, R_{\alpha \beta \mu \nu}\right)$ is a transcendental function of all 14 invariants of the Riemann tensor and is intractable. We follow the Einstein's observation: the Lagrangians of all known field theories are the simplest possible ones. In NLG theories, this rule is broken from the definition, nevertheless, we believe that the physical content (and tenability) of these theories may be established by studying classes of simple Lagrangians. In classical mechanics and field theory, the power of the canonical formalism lies in that physical Lagrangians are quadratic in "velocities". Accordingly, we assume that $L$ is a sum of 3 functions, each depending on one of the irreducible parts of the curvature tensor

$$
L=R+a R^{2}+b S_{\mu \nu} S^{\mu \nu}+f(W),
$$


where $S_{\mu \nu}=R_{\mu \nu}-\frac{1}{4} g_{\mu \nu} R$ is the traceless part of Ricci tensor and $W=$ $C_{\alpha \beta \mu \nu} C^{\alpha \beta \mu \nu}$ is the quadratic invariant of the Weyl tensor. We assume that $f$ is a simple function of $W$.

The analysis of the particle content of $L$ as in (1) is based on the fundamental property of all known field theories: each physical DOF corresponds to an independent (free) physical field in a multiplet. This means the following. Let a unifying field $\psi$ be decomposed into a multiplet of interacting fields $\phi_{1}, \ldots, \phi_{n}$. Let $\phi_{2}, \ldots, \phi_{n}$ be in their ground states, $\phi_{2}=\phi_{20}, \ldots, \phi_{n}=\phi_{n 0}$, then the full system of field equations for the multiplet is reduced to a subsystem for the independent free field $\phi_{1}$ carrying $F_{1}$ DOF. The same should hold for $\phi_{2}, \ldots, \phi_{n}$ and the sum $F_{1}+F_{2}+\ldots+F_{n}$ should be equal to the number $F$ of DOF for $\psi$. It is crucial that each field of the multiplet may be excited whereas the other fields are absent.

This is not the case of NLG theories: the fields of the resulting multiplet are connected by various relationships and some of them are devoid of independent existence. If the Weyl term $f(W)$ in $(1)$ is absent, one gets a triplet $\left\{g_{\mu \nu}, \pi^{\mu \nu}, \chi\right\}$, where $\chi$ is a momentum conjugate to $R$ and $\pi^{\mu \nu}$ is a momentum conjugate to $S_{\mu \nu}$. The field $\chi$ is coupled to $\pi^{\mu \nu}$ and cannot exist without it, in consequence, it is unphysical and should be removed from the Lagrangian and this is achieved by a special choice of the coefficients $a$ and $b$ in (1) [1]. Only the doublet $\left\{g_{\mu \nu}, \pi^{\mu \nu}\right\}$ carrying 7 DOF is tenable.

If the Weyl term $f(W)$ is present in (1), one arrives at the proposition: whether or not the $S_{\mu \nu}$ term is present in the Lagrangian $(b \neq 0$ or $b=0)$, the Weyl tensor contribution does not give rise to a well-behaved multiplet of gravitational fields. In other terms, any momentum conjugate to Weyl tensor (there are two possibilities) cannot exist independently as it may be excited only as a field interacting with the metric and then both the fields form a system with underdetermined evolution. In conclusion, from the purely field-theory viewpoint, the Weyl tensor should not appear in the Lagrangian of any NLG theory, only $L=f\left(g_{\mu \nu}, R, R_{\alpha \beta}\right)$ are admissible with various restrictions. In our opinion, this outcome strengthens the distinguished role of GR in the space of gravity theories.

\section{REFERENCES}

[1] G. Magnano, L.M. Sokołowski, "On the Lagrangian Structure of Metric Nonlinear Gravity Theories: the Redundant Weyl Tensor Dependence", in preparation.

[2] R. Penrose, W. Rindler, Spinors and Space-time, vol. 1, Two-spinor Calculus and Relativistic Fields, Cambridge Univ. Press, Cambridge 1984, par. 5.11. 